\title{
Selected abstracts from the Critical Care Canada Forum 2014
}

\author{
October 29 - November 1, 2014, Toronto, Ontario
}

\section{IMPROVING AN ICU DAILY GOALS CHECKLIST: INTEGRATED AND END-OF-GRANT KNOWLEDGE TRANSLATION}

Centofanti, John'; Duan, Erick²; Hoad, Neala ${ }^{3}$; Swinton, Marilyn²; Perri, Dan ${ }^{3}$; Waugh, Lily ${ }^{3}$; Soth, Mark ${ }^{3}$; Cook, Deborah ${ }^{3}$

${ }^{1}$ Anesthesia \& Critical Care, McMaster University; ${ }^{2}$ Critical Care, McMaster University; ${ }^{3}$ Critical Care, St Joseph's Healthcare Hamilton, Hamilton, Ontario

INTRODUCTION: Care of critically ill patients is dependent on a team of multidisciplinary clinicians working collaboratively. Although substantial communication occurs during morning ICU rounds, many factors jeopardize clear patient care communication and optimal patient care. In February 2012, a daily goals checklist (DGC) was introduced at our tertiary care, university-affiliated medical-surgical ICU for use during morning rounds.

OBJECTIVES: To understand the perspectives and attitudes of ICU clinicians about use of the DGC, then to improve the checklist by utilizing feedback from front line clinicians.

METHODS: We utilized a mixed-methods design. (1) Field Observations: 2 investigators conducted field observations to understand how the DGC was used for $80 \mathrm{ICU}$ patient rounds over 6 days. (2) Document Analysis: 72 completed DGCs from observed rounds were analyzed. (3) Interviews: We conducted semi-structured interviews of 56 clinicians, analyzing transcripts using a qualitative descriptive approach and content analysis. Triangulation was achieved by a multidisciplinary investigative team using 2 research methods and 3 data sources. Incorporating the feedback obtained from qualitative interviews using integrated knowledge translation (KT), we revised the DGC. We performed member checking at a multidisciplinary Quality Council meeting, intensivists' meeting, and with bedside nurses on site. As end-of-grant KT, we presented our findings at several scientific meetings, locally and internationally.

RESULTS: Clinicians identified the DGC to be a multipurpose tool impacting on 3 main domains: communication, patient care and education. It enhanced multidisciplinary communication, identified new patient care issues, and prompted teaching opportunities on rounds. The DGC was completed for $93 \%$ of our observed rounds, and appeared to foster closed-loop communication between nurses and physicians. Through qualitative analysis of interviews and subsequent member checking, we identified 4 themes related to enhancing the DGC: purpose, content, function and format. Recommendations follow (1) Purpose: expand explicit goal-setting to as many patient domains as possible, and systematic followup of progress between rounds. (2) Content: expand the physiotherapy section given the important role of rehabilitation in recovery. (3) Function: utilize the DGC to incorporate recent sedation guidelines. (4) Format: use phrases to facilitate critical thinking, and free text options for further context. Our KT activities led to ongoing collaboration at our own centre, and from other cardiac surgery and oncology ICUs locally.

CONCLUSION: The DGC at St Joseph's Healthcare Hamilton ICU is a locally developed tool that has helped to enhance communication, patient care and education. Integrated $\mathrm{KT}$ as the study was ongoing elicited ways to further enhance its use requiring periodic revision of purpose, content, function and format. End-of-grant KT generated feedback from front line clinicians for further modification before reintroducing the revised DGC into the ICU. Further study will be required to expand the DGC into other ICUs, and to assess its impact on patient-important outcomes.

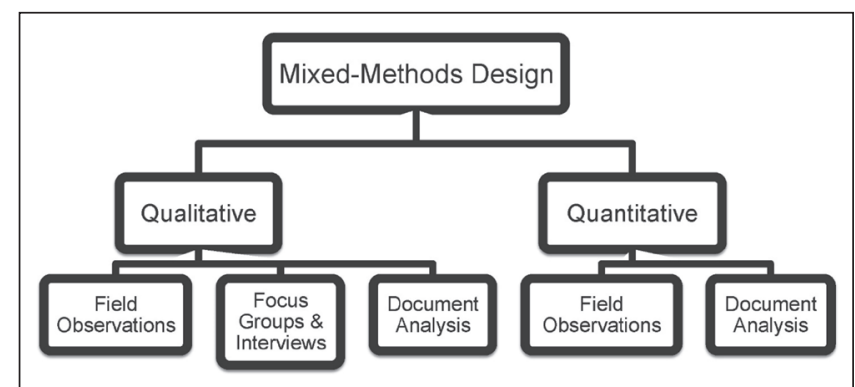

Figure 1) Schematic of study methods

\section{APPRAISAL OF A NOVEL MODEL TO IMPROVE PREDICTION OF EXTUBATION FAILURES IN CRITICALLY ILL PATIENTS \\ Westergaard, Paul ${ }^{1}$; Herry, Christophe ${ }^{2}$; Godard, Stephanie ${ }^{3}$; Scales, Nathan ${ }^{2}$; Seely, Andrew ${ }^{2}$ \\ ${ }^{1}$ Adult Critical Care Medicine Training Program, University of Ottawa; ${ }^{2}$ The Ottawa Hospital Research Institute, University of Ottawa; ${ }^{3}$ Faculty of Medicine, University of Ottawa, Ottawa, Ontario}

INTRODUCTION: Extubation failure (i.e. urgent re-intubation within 48-hrs) is associated with increased intensive care unit and hospital mortality, length of stay, tracheostomy rate, long term care requirements, and hospital costs. Spontaneous Breathing Trials (SBTs) are standard-of-care means to assess extubation readiness. However, despite passing an SBT with current performance measures, nearly $20 \%$ of patients fail extubation. Assessment of performance of SBTs is subjective and variable. SBT performance assessments require a more sensitive and objective model to predict extubation failure. OBJECTIVES: A novel multivariate predictive model (Weaning And Variability Evaluation (WAVE) Score) derived from respiratory rate variability (RRV) during the SBT prior to extubation, has previously demonstrated improved predictive accuracy of extubation outcomes compared with current standard measures of SBT performance. In this work, we examine the performance of WAVE Score in greater depth in order to better quantify the limitations and strengths of this tool.

METHODS: Data on 446 patients over 12 centers were taken from a prior study of patients utilized to derive the WAVE Score (performed with the Canadian Critical Care Trials Group). Subgroups for analysis were defined a priori based on clinical and physiological descriptors. A sensitivity analysis was performed by increasing and shortening the definition of extubation failure (i.e. varying the period of time before re-intubation), assessing impact on WAVE Score test performance. The evolution of WAVE Score performance during sequential SBTs prior to extubation was evaluated in 74 patients with 2 or more sequential SBTs recorded prior to extubation. RESULTS: The WAVE Score exhibits improved overall performance in ROC AUC and improved sensitivity for predicting extubation failure in specific subgroups of patients: patients who were less sedated with Richmond Agitation Sedation Scale (RASS) $>=-1$ (ROC AUC 0.75 vs. $0.58, \mathrm{p}=0.15$ ), or who were high risk patients with rapid shallow breathing index $(\mathrm{RSBI})>110$ (ROC AUC 0.93 vs. $0.70, \mathrm{p}=0.01$ ), or respiratory rate $(\mathrm{RR})>30$ (ROC AUC 0.85 vs. $0.65, \mathrm{p}=0.02$ ). Despite being derived from RRV, WAVE performance was not significantly altered in groups with a primary respiratory admission diagnosis or respiratory etiology for extubation failure. The WAVE Score demonstrated no significant performance variability when altering the definition of extubation failure (i.e. re-intubation in 12-72 hrs). In this observational study, the sequential WAVE Score over repeated SBTs demonstrated a stable low risk of extubation failure with a low average coefficient of variation of 0.09 . 
TABLE 1

Wave score subgroup analysis

\begin{tabular}{|c|c|c|c|c|c|c|c|c|c|}
\hline Subgroups & SEN & SPE & PPV & NPV & AUC & P-value (AUC) & $\begin{array}{c}\text { \# Failed } \\
\text { extubation }\end{array}$ & $\begin{array}{l}\text { \# Passed } \\
\text { extubation }\end{array}$ & Total \\
\hline $\mathrm{RSBI} \geq 110$ & 1.00 & 0.50 & 0.36 & 1.00 & 0.93 & 0.01 & 5 & 18 & 23 \\
\hline RSBI $<110$ & 0.71 & 0.59 & 0.18 & 0.94 & 0.70 & & 45 & 346 & 391 \\
\hline Age $\geq 60$ & 0.83 & 0.54 & 0.19 & 0.96 & 0.77 & 0.30 & 30 & 234 & 264 \\
\hline Age $<60$ & 0.62 & 0.63 & 0.19 & 0.92 & 0.68 & & 21 & 150 & 171 \\
\hline RASS $\geq-1$ & 0.78 & 0.58 & 0.20 & 0.95 & 0.75 & 0.15 & 41 & 305 & 346 \\
\hline RASS $<-1$ & 0.56 & 0.54 & 0.13 & 0.91 & 0.58 & & 9 & 76 & 85 \\
\hline $\mathrm{RR} \geq 30$ & 1.00 & 0.52 & 0.48 & 1.00 & 0.85 & 0.02 & 14 & 31 & 45 \\
\hline $\mathrm{RR}<30$ & 0.65 & 0.58 & 0.14 & 0.94 & 0.65 & & 37 & 346 & 383 \\
\hline
\end{tabular}

TABLE 2

Test characteristics of wave score RSBI, RR

\begin{tabular}{|c|c|c|c|c|c|c|c|c|}
\hline & & & & & AUCl & \#Failed & \# Passed & \\
\hline All patients & SEN & SPE & PPV & NPV & BACC* & extubation & extubation & Total \\
\hline $\begin{array}{l}\text { WAVE score } \\
\quad>0.5\end{array}$ & 0.75 & 0.58 & 0.19 & 0.94 & 0.72 & 51 & 385 & 436 \\
\hline $\mathrm{RSBI} \geq 110$ & 0.10 & 0.95 & 0.22 & 0.88 & $0.53^{*}$ & 50 & 364 & 414 \\
\hline$R R \geq 30$ & 0.27 & 0.92 & 0.31 & 0.90 & $0.60^{*}$ & 51 & 377 & 428 \\
\hline
\end{tabular}

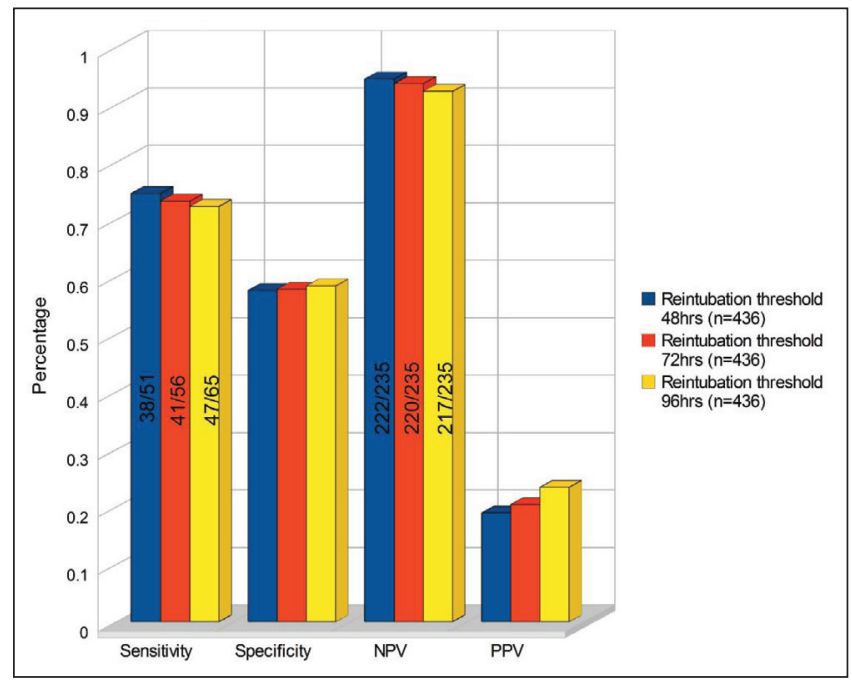

Figure 1) Sensitivity analysis: Prolonging re-intubation threshold

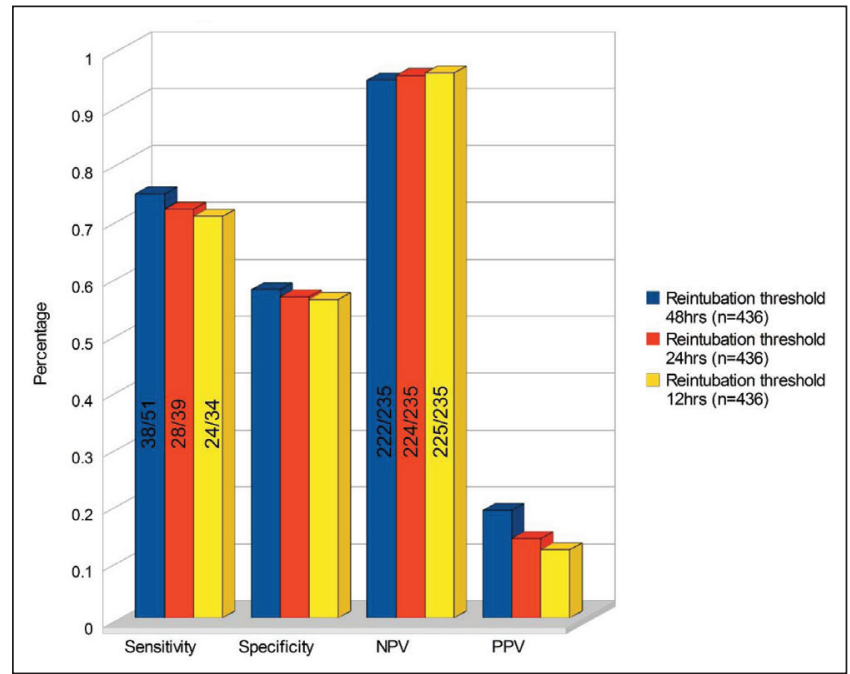

Figure 2) Sensitivity analysis: Reducing re-intubation threshold

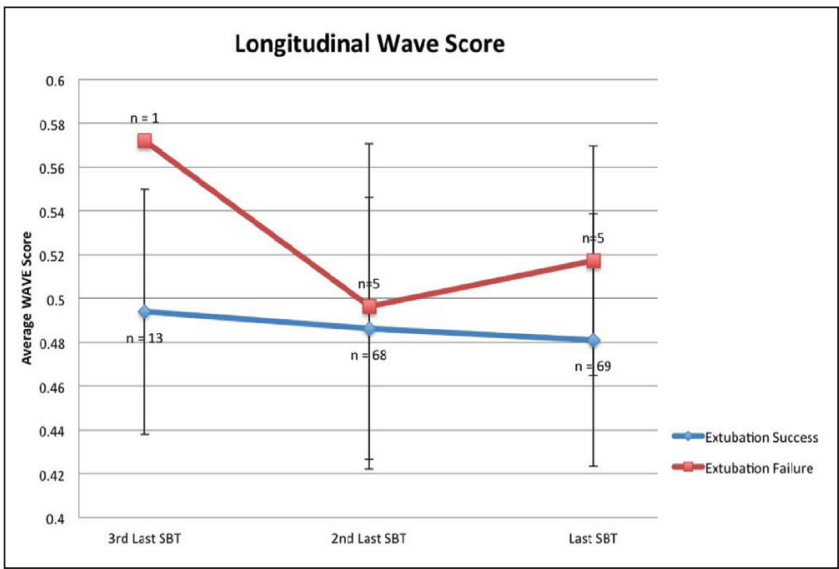

Figure 3) Longitudinal WAVE score: Can WAVE score predict earlier extubation?

CONCLUSION: The WAVE Score demonstrates improved performance, including sensitivity (ability to correctly detect extubation failure), in patients whom were less sedated (RASS $\geq-1$ ). While sedation is known to reduce RRV in general, these findings suggest sedation may also blunt the capacity of the WAVE score (due to reduced RRV) to detect inability to tolerate an increased workload of breathing during an SBT. The WAVE score performance appears to perform superiorly in patients with a high risk of failure based on elevated RSBI or RR. The WAVE Score demonstrated stable performance when changing the definition of time $(12-72 \mathrm{hrs})$ before reintubation. The clinical impact of providing the WAVE score to clinicians sequentially up to the time of extubation remains to be determined.

\section{REFERENCES}

1. Evidence-based guidelines for weaning and discontinuing ventilatory support: A collective task force facilitated by the American College of Chest Physicians; the American Association for Respiratory Care; and the American College of Critical Care Medicine.

Chest 2001;120(6 Suppl):375S.

2. Miu T, Joffe AM, Yanez ND, et al. Predictors of reintubation in critically ill patients. Respir Care 2014;59:2.

3. Ely EW, Baker AM, Evans GW, Haponik EF. The prognostic significance of passing a daily screen of weaning parameters. Intens Care Med 1999;25:581.

4. Esteban A, Frutos F, Tobin MJ, et al. A comparison of four methods of weaning patients from mechanical ventilation. Spanish Lung Failure Collaborative Group. N Engl J Med 1995;332:345.

5. Brochard L, Rauss A, Benito S, Conti G, Mancebo J, Rekik N, Gasparetto A, Lemaire F. Comparison of three methods of gradual withdrawal from ventilatory support during weaning from mechanical ventilation. Am J Respir Crit Care Med 1994;150:896. 


\section{CHARACTERIZATION OF PSYCHOTROPIC DRUG USE SURROUNDING PHYSICAL RESTRAINT APPLICATION IN MECHANICALLY VENTILATED, CRITICALLY ILL ADULTS}

Guenette, Melanie ${ }^{1}$; Farquharson, Tara $^{1}$; Cho, Ara $^{1}$; Wheeler, Kathleen ${ }^{1}$; Cheung, Alexandra ${ }^{1}$; Traille, Marlene ${ }^{2}$; Mantas, Ioanna ${ }^{1}$; Mehta, Sangeeta ${ }^{3}$; Rose, Louise ${ }^{4}$; Burry, Lisa ${ }^{1}$

${ }^{1}$ Pharmacy, Mount Sinai Hospital; ${ }^{2}$ Nursing, Mount Sinai Hospital, ; ${ }^{3}$ Medicine, Mount Sinai Hospital; ${ }^{4}$ Lawrence S Bloomberg Faculty of Nursing, University of Toronto, Toronto, Ontario

INTRODUCTION: Chemical restraint with psychotropic medications (e.g. benzodiazepines, non-benzodiazepine sedatives i.e., propofol and ketamine, opioids, and antipsychotics) is preferred over physical restraint (PR) for the management of agitation and for prevention of interference with medical devices. However, limited data exist describing the use of such drugs preceding and during PR application for critically ill adults.

OBJECTIVES: To characterize psychotropic drug use (i.e., alterations to drug regimens) both preceding and during PR application in critically ill, mechanically ventilated adults.

METHODS: Prospective single centre observational study of all patients physically restrained during invasive mechanical ventilation. Drug data were collected for three time intervals: 1) baseline, 120 to 61 minutes prior to $\mathrm{PR}$ application, 2) pre-PR, 60 to 0 minutes preceding PR application, and 3 ) post-PR, up to six hours after PR application. Types of psychotropic drug interventions (e.g. initiation, increase, decrease, and/or cessation) were recorded, as were the total time of PR use and Intensive Care Delirium Screening Checklist (ICDSC) scores.

RESULTS: Fifty-nine patients met inclusion criteria (31 male, 28 female), with a mean age of $59.5(\mathrm{SD}=18.7)$ years. Twenty-nine percent of patients screened positive for delirium, either during the nursing shift in which PR was applied, and/or the shift immediately following PR application. All patients were restrained using two-point Posey soft restraints, for a mean duration of $42.2(\mathrm{SD}=51.3)$ hours. During the pre-PR period, $16(27 \%)$ patients received no psychotropic drugs, 11 (19\%) had no changes to their existing drug regimen, and $32(54 \%)$ had a drug intervention. Twentyseven $(46 \%)$ patients had at least one drug initiated and/or increased in dosage during the pre-PR period, representing 41 prescriptions: $11(27 \%)$ opioids, 15 (37\%) benzodiazepines, two (5\%) antipsychotics, and $13(32 \%)$ non-benzodiazepine sedatives. During the post-PR period, five $(8 \%)$ patients continued to receive no psychotropic drugs, four $(7 \%)$ had no changes to their existing regimen, and 50 (85\%) had a drug intervention. Forty-two (71\%) patients had at least one drug initiated and/or increased in dosage during the post-PR period, representing 71 prescriptions: 29 (41\%) opioids, 19 (27\%) benzodiazepines, seven (10\%) antipsychotics, and $16(23 \%)$ non-benzodiazepine sedatives.

CONCLUSION: These data suggest that most patients receive psychotropic drugs immediately prior to, or early in the application of PR. Most interventions were new drug initiations, and/or increases in existing regimens, suggesting efforts are made to improve chemical restraint.

\section{HUMAN MESENCHYMAL STEM/STROMAL CELLS ENHANCE FCG RECEPTOR MEDIATED PHAGOCYTOSIS OF ESCHERICHIA COLI IN HUMAN MONOCYTE DERIVED \\ MACROPHAGES}

Garces-Ramirez, Linda

Anesthesia Research, Keenan Research Centre for Biomedical Science of St Michael's Hospital, University of Toronto, Toronto, Ontario

INTRODUCTION: Human Mesenchymal Stem/Stromal Cells (hMSCs) constitute a promising therapeutic strategy for sepsis and the Acute Respiratory Distress Syndrome. MSCs modulate the immune response to reduce lung injury, and enhance the clearance of bacteria, in murine and rodent Escherichia coli pneumonia (Gupta et al, 2012), and in the isolated human lung (Lee et al, 2013). The mechanisms by which MSCs exert beneficial effects are complex, and include their ability to modulate macrophage phenotype and function. A key function of macrophages (Mf) is to phagocytose and clear invading microorganisms.

OBJECTIVES: To explore if hMSCs are capable of enhancing IgG mediated FcgR Mf phagocytosis.
METHODS: Bone marrow hMSCs and peripheral blood monocytes were isolated from healthy donors. Monocytes were differentiated to Mf over 5 days. We assessed the ability of Mf, co-cultured for 3 days with hMSCs or fibroblasts, to ingest E. coli using two methods. In the first assay live $E$. coli was added to $\mathrm{Mf}$ and 15 minutes later extracellular bacteria were washed. Intracellular E. coli, released from Mf by lysis, were plated and colonies (CFU) counted at $24 \mathrm{~h}$. In the second assay we tested Fcg receptormediated phagocytosis, by adding opsonized E. coli bioparticles (tagged with green fluorophore) to Mf. Cells were fixed with 4\% PFA, and streptavidin conjugate (tagged with red fluorophore) was added to enable visualization of extracellular bacteria. Images were captured using a confocal microscope. Counting of phagocytosed (green) or non-phagocytosed (red) E. coli was done at 7 randomly chosen fields/slide using Image-J software. RESULTS: In both assays, Mf co-cultured with hMSCs engulfed significantly more E. coli than Mf alone (control group) or Mf co-cultured with fibroblasts. In the first assay ( $\mathrm{n}=3) \mathrm{Mf}$ co-cultured with hMSCs (on inserts or directly) engulfed approximately $2 \times$ more live bacteria than Mf alone $(\mathrm{P}<0.001)$. Mf co-cultured directly in contact with hMSCs did not further enhance phagocytosis. Fibroblast co-culture had no effect on Mf phagocytosis. Our results, therefore, suggest that factors secreted from hMSCs enhance Fcg receptor mediated Mf phagocytosis.

CONCLUSION: Our study has shown that hMSCs enhance FcgR Mf phagocytosis. Further experiments will explore the effects of hMSCs on other phagocytic pathways.

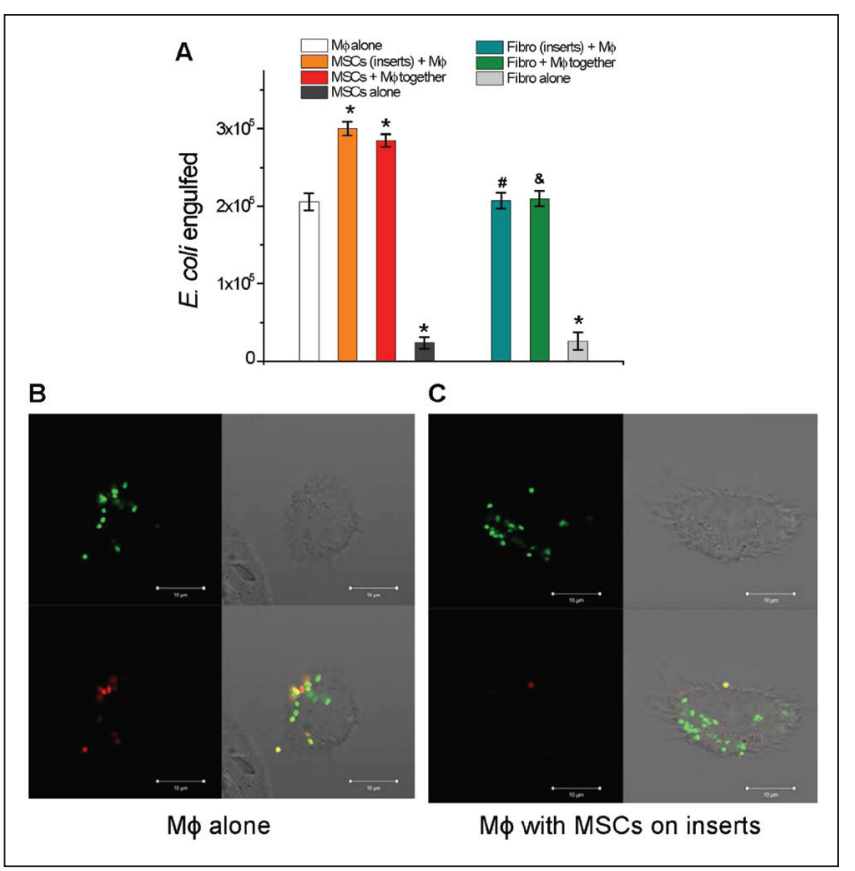

Figure 1) $h M S C$ s enhance $F_{c y} R$ M $\phi$ phagocytosis A E. coli phagocytosis is increased when $\mathrm{M} \phi$ are co-cultured with MSCs (on insert or directly) but not with fibroblasts; $N=4$ experiments ${ }^{*} P<0.05$ vs. $\mathrm{M} \phi$ alone group, ${ }^{\#} P<0.05$ vs. MSCs (or insert) $+M \phi$ group, ${ }^{\mathcal{B}} \mathrm{P}<0.05$ vs. MSCs $+\mathrm{M} \phi$ (together) group. Bar represents mean \pm SEM. B Representative image of $M \phi$ alone group showing that approximately half of E. coli (green) appears to be extracellular (red). C Representative image of MSCs (on insert) $+M \phi$ group showing that almost all bacteria is engulfed by $\mathrm{M} \phi$.

\section{REFERENCES}

1. Gupta N, Krasnodembskaya A, Kapetanaki M, Mouded M, Tan X, Serikov V, Matthay MA. Mesenchymal stem cells enhance survival and bacterial clearance in murine Escherichia coli pneumonia. Thorax 2012;67:533-539. doi: 10.1136/thoraxjnl-2011-201176.

2. Lee JW, Krasnodembskaya A, McKenna DH, Song Y, Abbott J, Matthay MA. Therapeutic effects of human mesenchymal stem cells in ex vivo human lungs injured with live bacteria. Am J Respir Crit Care Med 2013;187:751-760. doi: 10.1164/rccm.201206-09900C. 


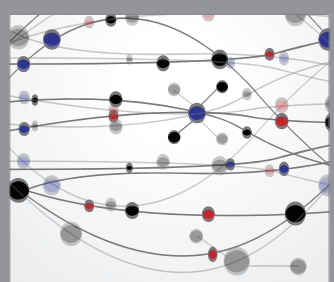

The Scientific World Journal
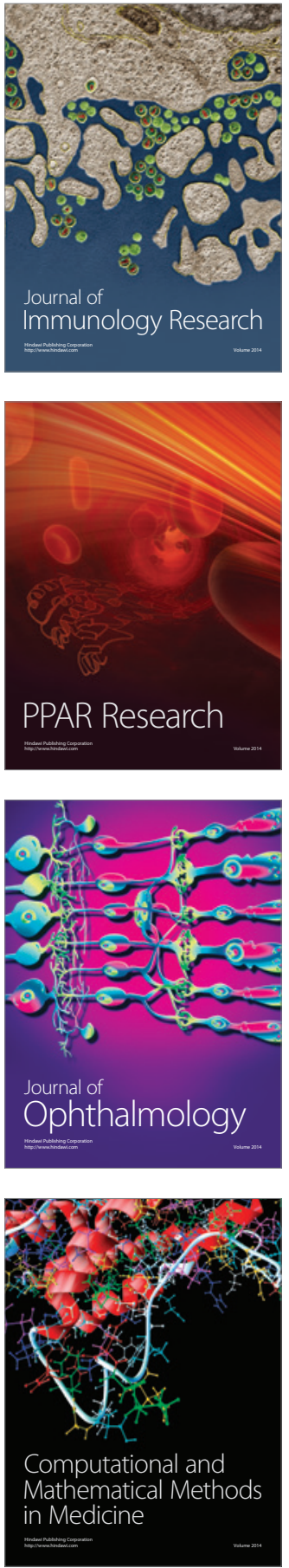

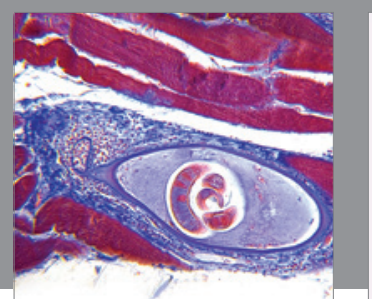

Gastroenterology Research and Practice

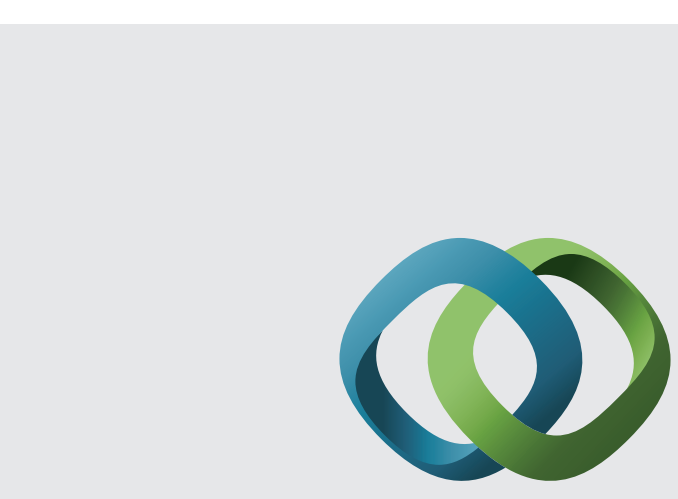

\section{Hindawi}

Submit your manuscripts at

http://www.hindawi.com
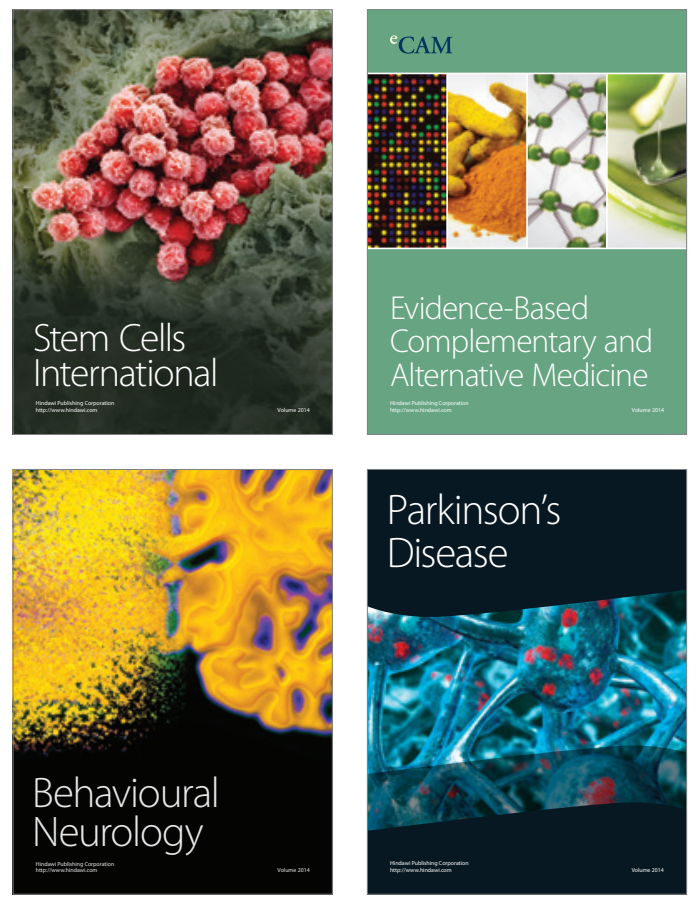
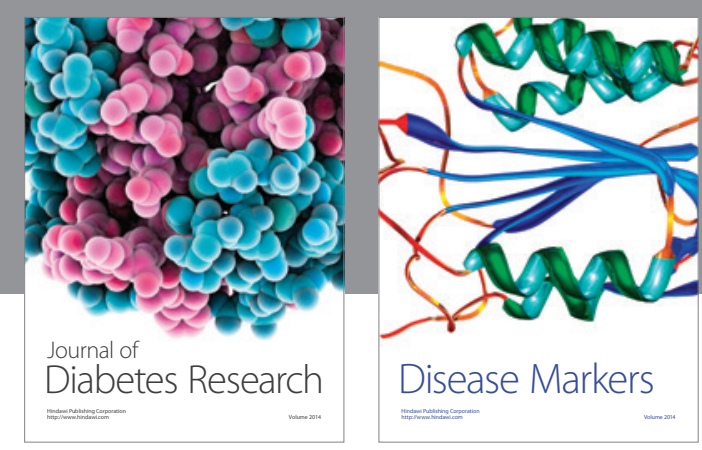

Disease Markers
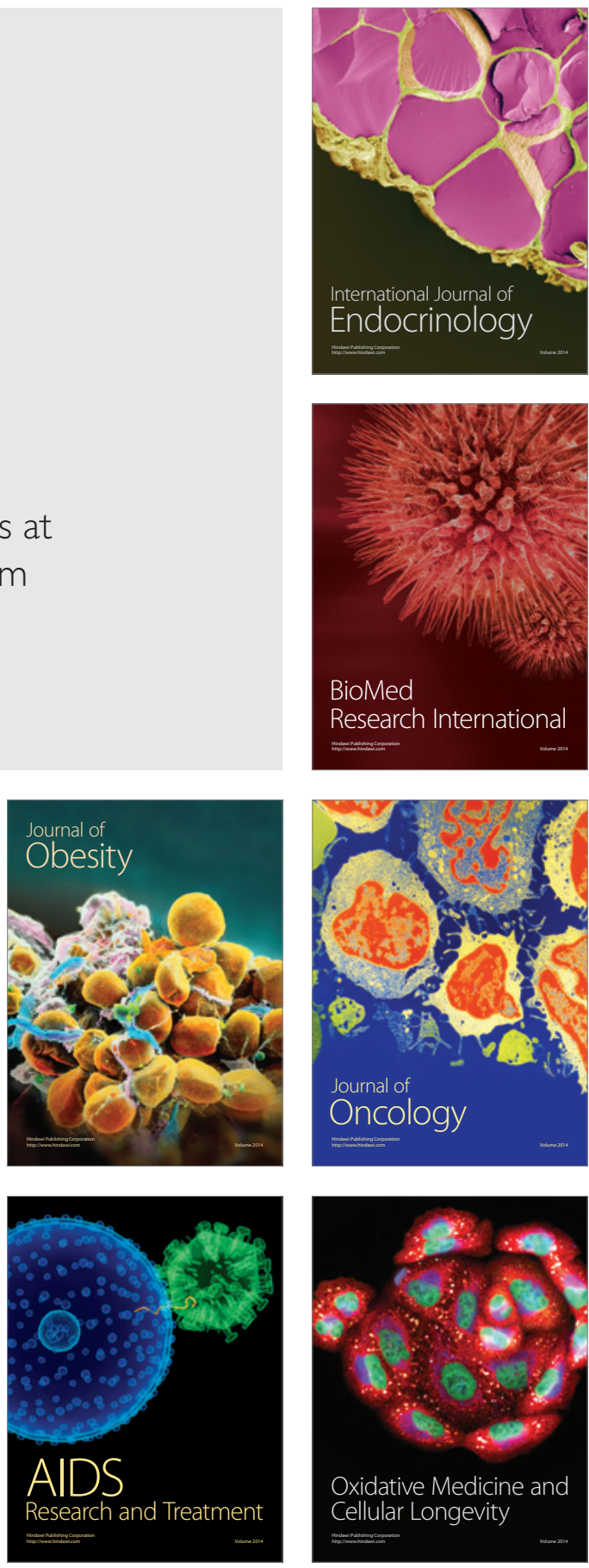